\title{
Toll-like receptor 4-mediated immune stress in pregnant rats activates STAT3 in the fetal brain: role of interleukin- 6
}

\author{
Abdeslam Mouihate $^{1}$ and Heba Mehdawi ${ }^{1}$
}

\begin{abstract}
BACKGROUND: Prenatal exposure to pathogens induces long lasting effect on brain function and plasticity. It is unclear how maternal immune stress impacts fetal brain development. Immune challenged pregnant rats induce the production of inflammatory cytokines including tumor necrosis factor (TNF) $\alpha$, interleukin (IL)1 $\beta$, and IL-6. IL-6 crosses the placenta but its mechanism of action on fetal brain is unclear.
\end{abstract}

METHODS: Gestation day 15 (GD15) rats were given a single injection of lipopolysaccharide (LPS) $(100 \mu \mathrm{g} / \mathrm{kg})$ in the presence or the absence of an IL-6 neutralizing antibody (IL-6Ab, 10 $\mu \mathrm{g} / \mathrm{kg}$ ). The activation of the intracellular signal of IL-6; signal transducer and activator of transcription (STAT3) and levels of glucocorticoids (GCs) were monitored in fetal brains.

RESULTS: LPS administration to GD15 rats significantly increased the phosphorylation levels of STAT3 in fetal brains. Such activation was blunted by IL-6Ab. LPS induced a significant rise in GCs in the plasma of dams but not in fetal brains. IL-6Ab significantly reduced LPS-induced GCs in maternal plasma.

CONCLUSION: Toll-like receptor 4 (TLR4)-induced activation of the maternal innate immune system affects fetal brains likely via the mobilization of IL-6/STAT3 pathway. In contrast, TLR4stimulated maternal GCs release is less likely to play a significant role in fetal brain development.

$\mathbf{E}_{\mathrm{le}}^{\mathrm{x}}$ posure to viral or bacterial pathogens during pregnancy leads to enhanced risk of psychiatric disorders in developing and adult offspring (1). Experimentally, adult offspring of rodents exposed to either bacterial or viral active agents during pregnancy show neurological disturbances, including enhanced hypothalamo-pituitary-adrenal axis activity associated with depression-like behavior and heightened stress response $(2,3)$, altered brain plasticity and increased risk to develop schizophrenia-like behavior (4). While it is well established that prenatal immune system activation is associated with many brain-associated illnesses during adulthood, few studies have addressed the mechanism through which maternal immune system alters fetal brain development. It is largely accepted that the maternal immune system response to pathogens, rather than the pathogens themselves, negatively impacts fetal brain development and thus contribute to these long lasting deleterious effects (5).
Experimentally, prenatal immune challenge is mimicked by a systemic injection of lipopolysaccharide (LPS), the active ingredient of Gram-negative bacteria, to pregnant rodents. Maternal immune competent cells, including peritoneal macrophages and spleen cells, release a series of proinflammatory cytokines; the most important of which are TNF- $\alpha$, IL-1 $\beta$, and IL-6 (5). These proinflammatory cytokines could potentially affect fetal brain development. However, whether maternally-borne proinflammatory cytokines enter the fetal compartment and directly affect fetal brain development is still debatable $(4,5)$.

Evidence exist to suggest that, among these proinflammatory cytokines, relatively large amounts of IL- 6 cross the placenta (6), access fetal brain and thus could affect its developmental trajectory leading to long lasting effect on brain plasticity and function. The intracellular signaling pathway of IL-6 involves its binding to the IL- 6 receptor leading to the phosphorylation of the intracellular signal transducer and activator of transcription (STAT3) (7). Additionally, IL-6 could indirectly affect the fetal brain development through its stimulatory effect on glucocorticoids (GCs) release and the negative impact of the latter on neural cell death and survival (8).

In the present study, we tested whether maternal immune challenge with LPS affects IL-6-dependent STAT3 activation in fetal rat brains and assessed the role of IL- 6 in corticosterone (rat's major GC) mobilization both in maternal circulation and fetal brain tissue. We give evidence that maternal exposure to LPS during pregnancy activates STAT3 in the fetal brain in an IL-6-dependent manner. Furthermore, LPS-induced mobilization of maternal corticosterone was not associated with enhanced levels of corticosterone in fetal brains.

\section{RESULTS}

\section{Maternal Side}

LPS induced an enhanced expression of phosphorylated form of p-STAT3 in maternal spleen in the absence of IL-6Ab (Figure 1a,b). LPS administration did not significantly enhance p-STAT3 expression levels when coinjected with the IL-6Ab. Compared to the LPS-induced increase in p-STAT3 levels seen in the absence of IL-6Ab, injection of IL-6Ab significantly reduced LPS-enhanced expression of p-STAT3 in maternal spleen. The coadministration of IL-6Ab with LPS significantly reduced IL-6 levels in maternal plasma (Figure 1c). 


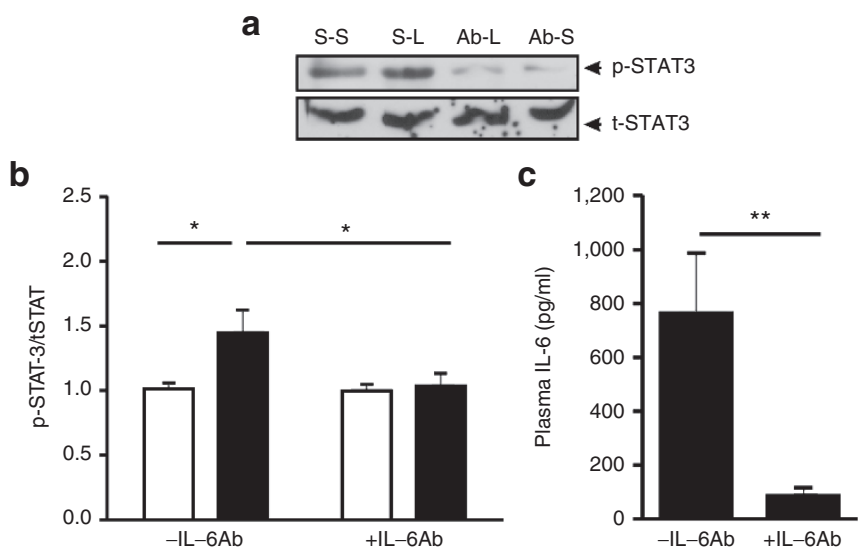

Figure 1. IL-6 neutralizing antibody dampens lipopolysaccharide (LPS) activated STAT3 in maternal spleen. (a) Western blot detection of phosphorylated (p-STAT3) and total STAT3 (t-STAT3) proteins in the spleen of GD15 rats given either saline (S-S and Ab-S) or LPS (S-L and $A b-L)$, in the absence (S-S and S-L) or the presence of the IL-6Ab (Ab-L and Ab-S) $4 \mathrm{~h}$ after LPS injection. (b) Bar graph shows the levels of p-STAT3 in the spleen of GD15 rats given either saline (open bars) or LPS (solid bars) in the absence or the presence of the IL-6Ab. (c) Bar graph shows the IL-6 levels in the plasma of GD15 rats given LPS in the presence or the absence of the IL-6Ab. For p-STAT3 data: (Saline (-IL-6Ab; $n=6$ ), LPS (-IL-6Ab; $n=6$ ), Saline $(+\mathrm{IL}-6 \mathrm{Ab} ; n=6)$, LPS (+IL-6Ab; $n=6))$. For plasma IL-6 data $((-\mathrm{IL}-6 \mathrm{Ab}, n=5)$, $(+\mathrm{IL}-6 \mathrm{Ab}, n=4)) .{ }^{*} P<0.05,{ }^{* *} P<0.01$. STAT3, signal transducer and activator of transcription.

LPS-induced IL-6 can stimulate corticosterone release $(9,10)$ which could account for the altered fetal brain development (11). Thus, we analyzed the impact of IL-6Ab on LPS stimulated corticosterone in maternal plasma. In the absence of IL-6Ab, LPS induced a significant increase in the levels of plasma corticosterone in GD15 rats (Figure 2). This enhanced corticosterone levels were also observed when LPS was coinjected with IL-6Ab. However, the LPS-induced enhanced levels of maternal plasma corticosterone were significantly reduced when IL-6Ab was coinjected with LPS.

\section{Fetal Side}

Figure 3a shows that in the absence of IL-6Ab, LPS induced a significant increase in the phosphorylated levels of STAT3 in the fetal brains at $4 \mathrm{~h}$ post-LPS injection. LPS administration to GD15 rats did not significantly enhance p-STAT3 levels in the fetal brains when coinjected with the IL-6Ab. Compared to the LPS-induced increase in p-STAT3 levels seen in the absence of IL-6Ab, injection of IL-6Ab significantly reduced the LPS-enhanced expression of p-STAT3 in fetal brains. Such inhibitory effect of IL-6Ab on LPS-enhanced p-STAT3 in fetal brains was absent when LPS was coinjected with a goat IgG (Figure 3c).

Similar effects of IL-6Ab where seen when the levels of p-STAT3 were measured $24 \mathrm{~h}$ after LPS injection to GD15 rats (Figure $3 b$ ). It is noteworthy that the levels of p-STAT3 were significantly lower at $24 \mathrm{~h}$ post-LPS injection when compared to their nadir values seen at $4 \mathrm{~h}$ post-LPS injection.

There was no significant effect of LPS on corticosterone levels in the fetal brain at both 4 and $24 \mathrm{~h}$ post-LPS injection (Figure 4). It is noteworthy that there was a significant decline

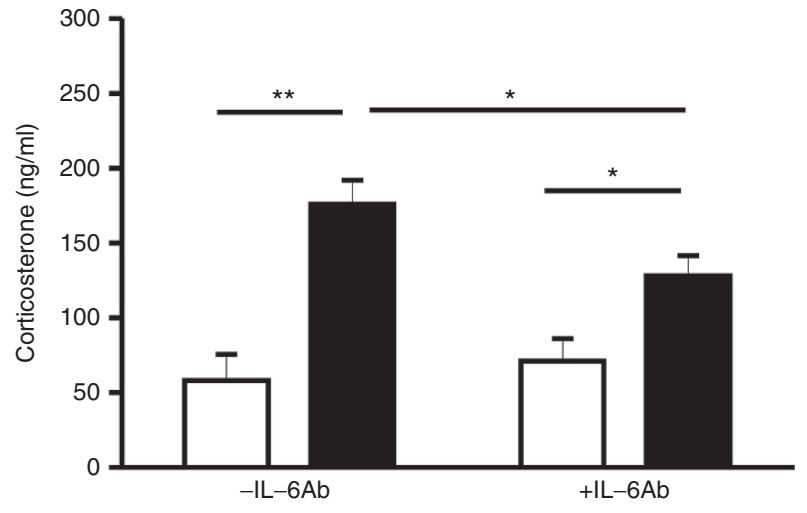

Figure 2. IL-6 neutralizing antibody decreases lipopolysaccharide (LPS)induced plasma corticosterone in GD15 rats. GD15 rats were given either saline (open bars) or LPS (solid bars) in the absence or the presence of IL-6Ab. Four hours later, their blood was collected and plasma corticosterone levels were assessed using ELISA. In the absence of IL-6Ab, LPS induced a strong increase in corticosterone levels in maternal plasma. Such LPS-induced increase in corticosterone levels was significantly reduced when GD15 rats were injected with the IL-6Ab. Saline (-IL-6Ab; $n=7)$, LPS (-IL-6Ab; $n=6)$, Saline (+IL-6Ab; $n=8)$, LPS (+IL-6Ab; $n=8)$, ${ }^{*} P<0.05,{ }^{* *} P<0.001$.

in the levels of corticosterone at $24 \mathrm{~h}$ post-LPS injection when compared to those seen at $4 \mathrm{~h}$ post-LPS injection.

Prenatal LPS has been associated with neural cell death by apoptosis $(12,13)$. One of the hallmark of neural cells committed to apoptosis is their expression of activated caspase 3 (14). To assess whether an immune challenge during pregnancy alters neural cell death in fetal brains, we explored the effect of LPS injection to GD15 rats on the number of activated caspase 3 in the fetal brains at 4 and $24 \mathrm{~h}$ post-LPS injection. Figure $5 \mathrm{a}$ shows a micrograph of a fetal brain with neural cells immunopositive to activated caspase 3 . Serial count of activated caspase 3-containing cells in both the parenchymal (Inside) and the outer-layer of the fetal brain (Edge) showed that neural cell death occurs naturally during fetal brain development (see fetal brains of saline injected GD15 rats). LPS injection, in the absence or the presence of IL-6Ab, did not significantly affect neural cell death in the rat fetal brains at either $4 \mathrm{~h}$ (Figure $5 \mathrm{~b}, \mathrm{c}$ ) or $24 \mathrm{~h}$ after the immune insult to GD15 dams (Figure 5d,e).

Double immunofluorescent staining was performed in fetal brains and showed that STAT3 protein colocalized with the astrocytic marker glial fibrillary acidic protein but not with doublecortin; a marker of newly born neurons (Figure 6a,b).

\section{DISCUSSION}

A series of novel data presented in the present paper support the following: (i) LPS-induced activation of the maternal immune system during pregnancy activates STAT3 in the fetal brain, (ii) LPS-activated STAT3 in maternal spleen and fetal brain is largely mediated by IL- 6 because this effect was significantly blunted by an antibody-based neutralization of IL-6, (iii) LPS induced an increase in maternal plasma corticosterone levels which was partly mediated by IL-6. In the fetal brain, the corticosterone levels were not significantly enhanced by maternal exposure to LPS, (iv) maternal exposure to LPS during pregnancy did not 
a

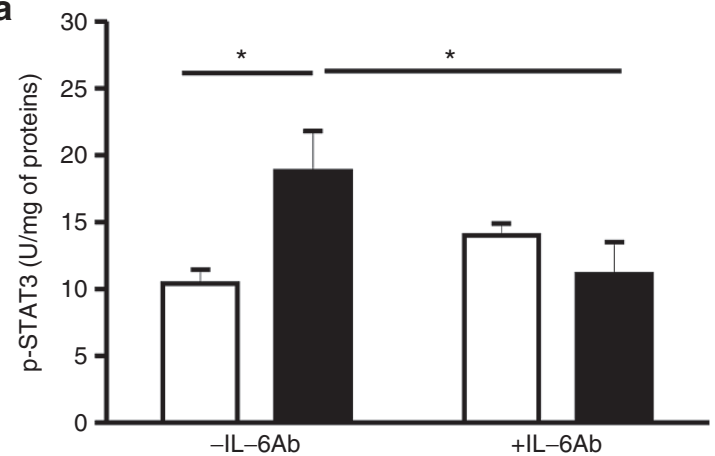

b

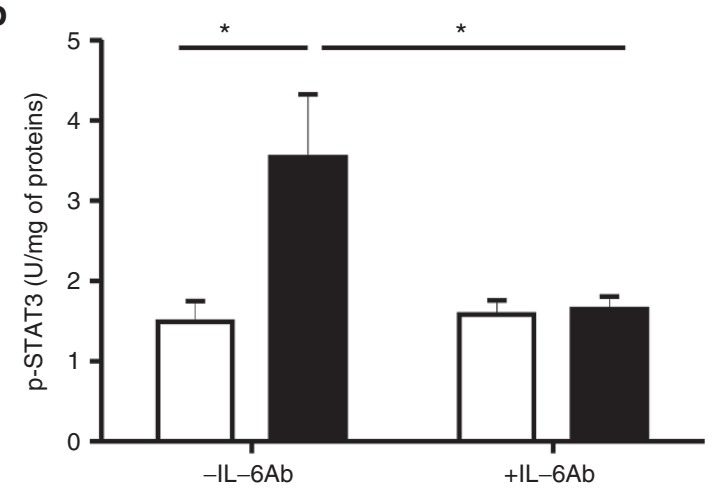

C

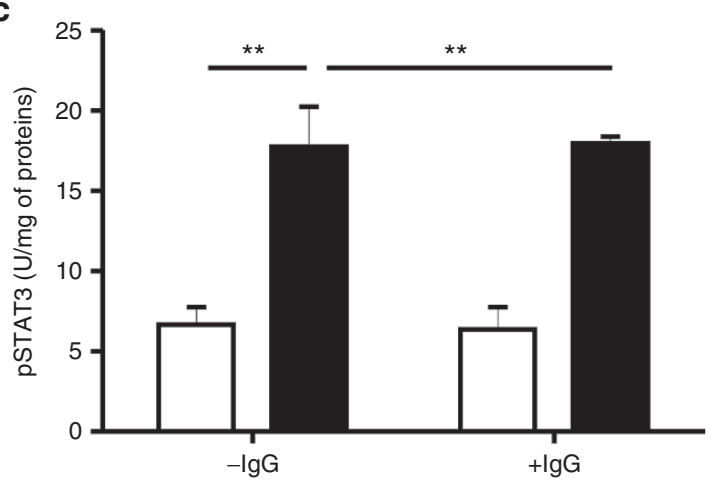

Figure 3. IL-6 neutralizing antibody blunts lipopolysaccharide (LPS)-induced p-STAT3 in fetal brains. GD15 rats were given either saline (open bars) or LPS (solid bars) in the absence (-IL-6Ab) or the presence of IL-6Ab (+IL-6Ab). Their fetuses were harvested at either $4 \mathrm{~h}$ (panel a) or $24 \mathrm{~h}$ (panel b) after LPS injection. Levels of $p$-STAT3 in the fetal brains were assessed using ELISA. In the absence of IL-6Ab, LPS induced a significant increase in p-STAT3 at both 4 and $24 \mathrm{~h}$ postinjection. Such LPS-induced increase in p-STAT3 levels was blunted in the brains of fetuses derived from dams injected with the IL-6Ab at 4 and $24 \mathrm{~h}$ post-LPS injection. Panel (c) shows the impact of LPS on the phosphorylation levels of STAT-3 in fetal brains in the presence (+lgG) or the absence (-lgG) of goat immunoglobulin $4 \mathrm{~h}$ after LPS injection. Saline (-IL-6Ab/4h, $n=6)$, LPS (-IL-6Ab/4h, $n=6)$, Saline (+IL-6Ab/4h, $n=6$ ), LPS (+IL-6Ab/4h, $n=7$ ), Saline $(-\mathrm{IL}-6 \mathrm{Ab} / 24 \mathrm{~h}, n=5)$, LPS (-IL-6Ab/24h, $n=5)$, Saline (+IL-6Ab/24h, $n=6)$, LPS (+IL-6Ab/24h, $n=5)$, Saline (-lgG, $n=5)$, Saline (+lgG, $n=5), \mathrm{LPS}(-\operatorname{lgG}, n=3)$, LPS $\left.(+\operatorname{lgG}, n=3) .{ }^{*} P<0.05,{ }^{* *} P<0.01\right)$. STAT3, signal transducer and activator of transcription.

result in overt neural cell death in fetal brains, as monitored by the amount of neural cells expressing cleaved caspase- 3.

Maternal Immune Stress and IL-6-STAT3 Signaling in Fetal Brain Several studies have shown that IL-6 protein levels are enhanced in fetal brain of dams given LPS (15). But the source

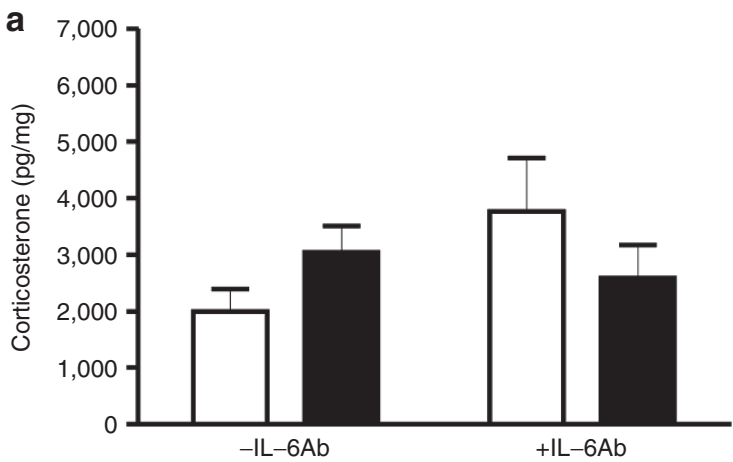

b

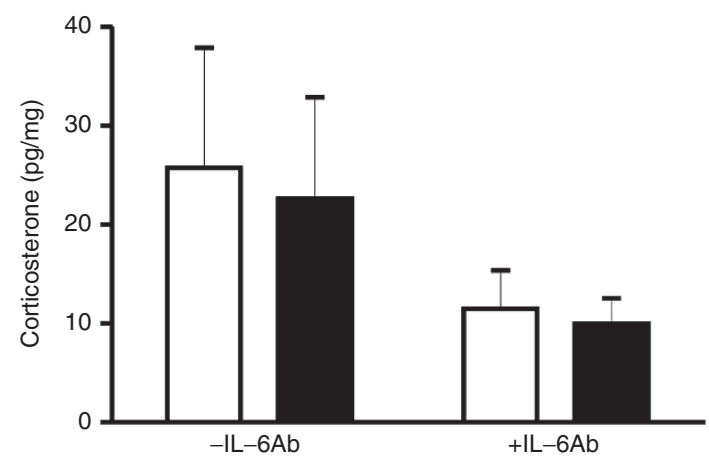

Figure 4. Lipopolysaccharide (LPS) injection to GD rats does not affect corticosterone levels in the fetal brain. GD15 rats were given either saline (open bars) or LPS (solid bars) in the absence (-IL-6Ab) or the presence of IL-6Ab (+IL-6Ab). Their fetuses were harvested at either $4 \mathrm{~h}(\mathrm{a})$ or $24 \mathrm{~h}$ (b) after LPS injection. Injection of LPS and/or IL-6Ab to GD15 rats did not significantly affect the amounts of corticosterone in the fetal brains at either 4 or $24 \mathrm{~h}$ post LPS injection. Saline (-IL-6Ab/4h, $n=7)$, LPS (-IL-6Ab/4h, $n=6)$, Saline (+IL-6Ab/4h, $n=6)$, LPS (+IL-6Ab/4h, $n=7)$, Saline (-IL-6Ab/24h, $n=5)$, LPS (-IL-6Ab/24h, $n=6)$, Saline (+IL-6Ab/24h, $n=6)$, LPS (+IL-6Ab/24h, $n=5)$.

of this IL-6 is not well-established. There are evidence to suggest that maternally borne IL-6 preferentially crosses the maternal-fetal placenta to reach fetal brain through an immature blood-brain barrier (16). Alternatively, the placenta can be the target of bacterial and viral pathogens and thus could form a potential source of IL-6 $(17,18)$. However, IL-6 mRNA levels were also shown to significantly increase within the fetal brain, suggesting a local synthesis of this cytokine within the fetal brain (19). Regardless of the IL-6 source, the present data show that maternal Toll-like receptor 4 (TLR4) stimulation leads to STAT3 activation in fetal brain in an IL-6-dependent manner.

It is noteworthy that STAT3 can also be activated by several other cytokines such as leukemia inhibitory factor, bone morphogenic protein 2, or leptin (20). However, the observation that LPS activated fetal STAT3 was blunted in the presence of the IL-6Ab suggests that these other STAT3 activators play a minimal role in the LPS-activated STAT3 in fetal brain. In addition to TLR4, previous studies have shown that TLR3 activation (by the viral mimetic Polyinosinic:polycytidylic acid) of the maternal innate immune system induced developmental and behavioral alteration in adult offspring (21). These TLR3-triggered alterations were largely attributed to IL-6 (22). Taken together, the proinflammatory cytokine IL-6 appears to 


\section{Articles $\mid$ Mouihate and Mehdawi}

a

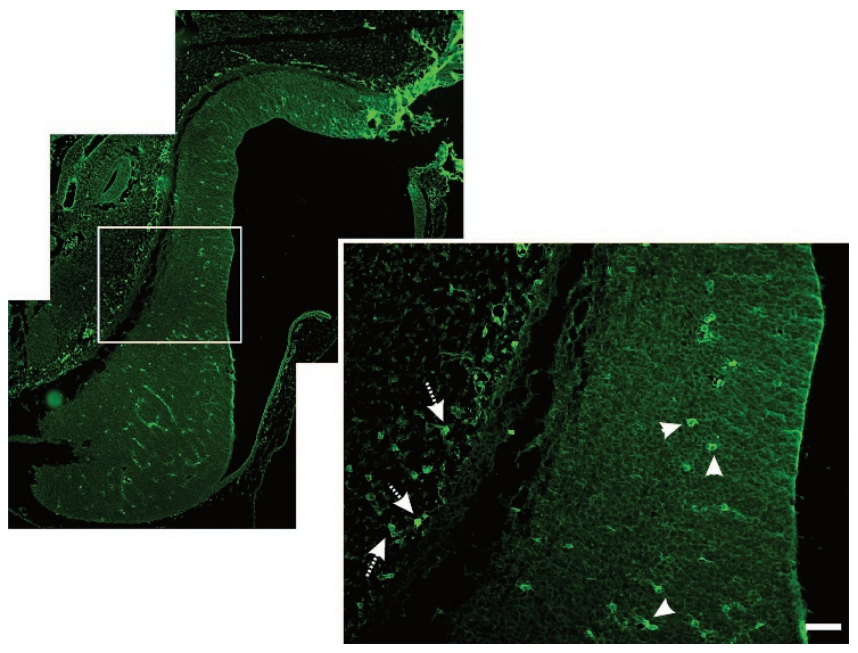

b

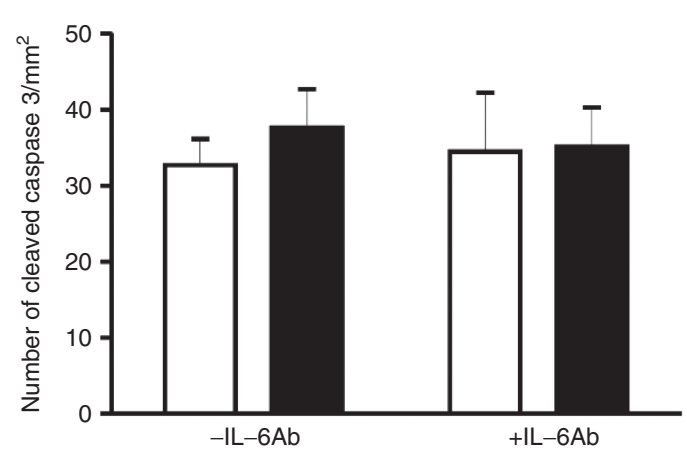

d

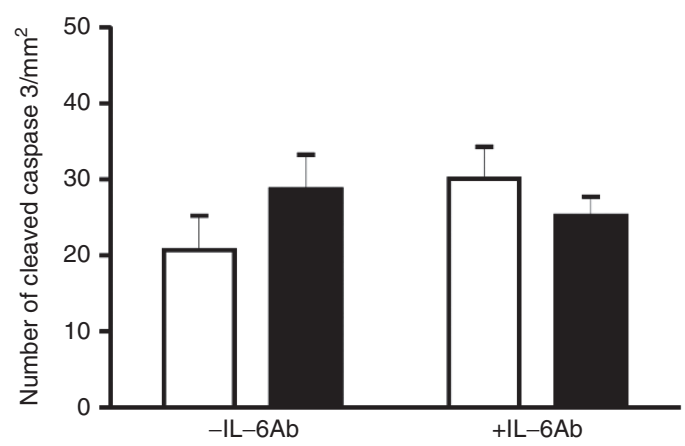

C

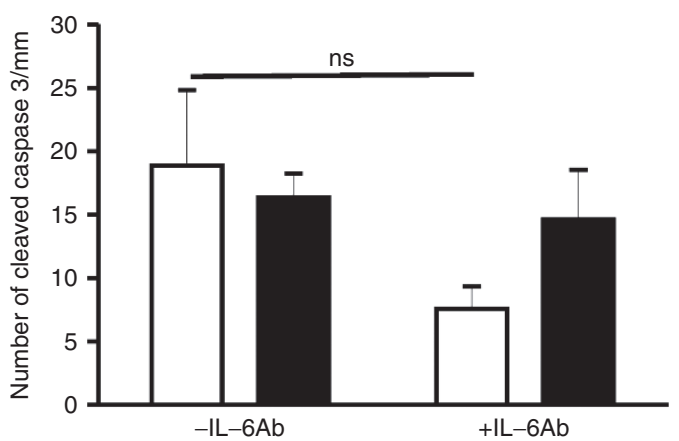

e

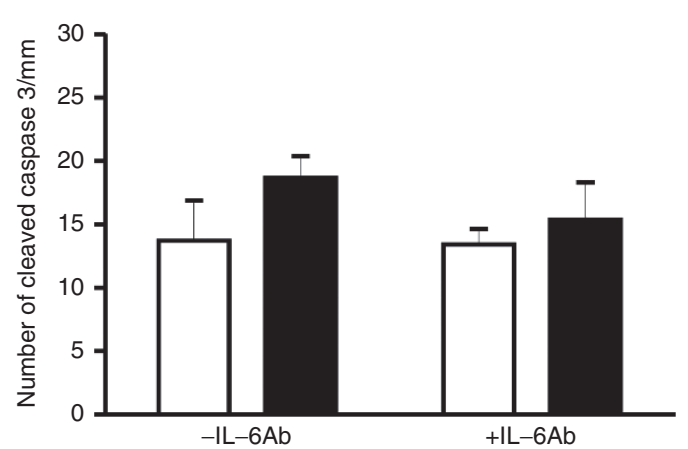

Figure 5. Lipopolysaccharide (LPS) injection to GD15 rats does not affect neural cell death in the brains of their fetuses. (a) micrograph of a fetal brain section immunostained with an antibody antiactivated caspase 3. The right side of the micrograph shows a magnified picture of the area delimited by the white square shown on the left picture. The number of cells immunoreactive to activated caspase 3 were counted in the parenchymal area of the brain as indicated by the arrowhead in the micrograph (inside; $\mathbf{b}$ and $\mathbf{d}$ ) and on the border of the brain as indicated by the arrows in the micrograph (edge; $\mathbf{c}$ and $\mathbf{e}$ ) in the presence (solid bars) or the absence of LPS injection (opens bars). Injection of LPS and/or IL-6Ab to GD15 rats did not significantly affect the number of activated caspase 3-containing cells in the brains of their fetuses at both $4 \mathrm{~h}(\mathbf{b}, \mathbf{c})$ and $24 \mathrm{~h}(\mathbf{d}, \mathbf{e})$ postinjection. Saline (-IL-6Ab/4h, $n=5)$, LPS (-IL-6Ab/4h, $n=5)$, Saline (+IL-6Ab/4h, $n=8)$, LPS (+IL-6Ab/4h, $n=5)$, Saline (-IL-6Ab/24h, $n=5)$, LPS (-IL-6Ab/24h, $n=6)$, Saline (+IL-6Ab/24h, $n=6)$, LPS (+IL-6Ab/24h, $n=6)$. ns, not significant.

mediate the impact of both viral (TLR3) and bacterial (TLR4) pathogens on fetal brain development.

The mechanism through which the prenatal immune challenge affects fetal brain is not well-understood. There are indications that STAT3 gene could play a role in the switch between neurogenesis and gliogenesis during fetal brain development. Indeed, inactivation of the STAT3 gene leads to premature neurogenesis (23), while its activation is required for the emergence of astrocytes (24). In the present study, we found that
STAT3 was expressed mostly in glial fibrillary acidic protein containing cells but not in doublecortin containing cells (newly born neurons). These data are in agreement with a previous study where STAT3 was not expressed in neurons of mouse fetal brain (23). Furthermore, we have previously shown that activation of TLR4 at a neonatal period led to an enhanced number of astrocytes in a sub-region of the hippocampus during adulthood (25). Whether such sustained enhanced expression of astrocytes occurs in rats prenatally exposed to TLR4 
a
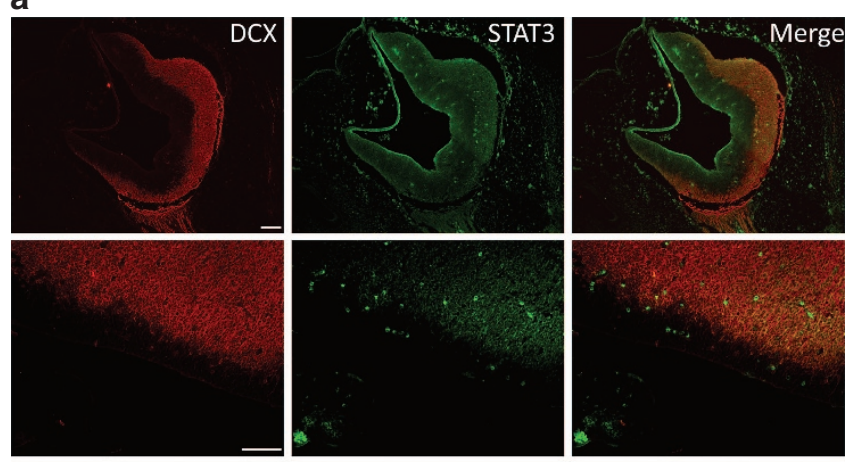

b
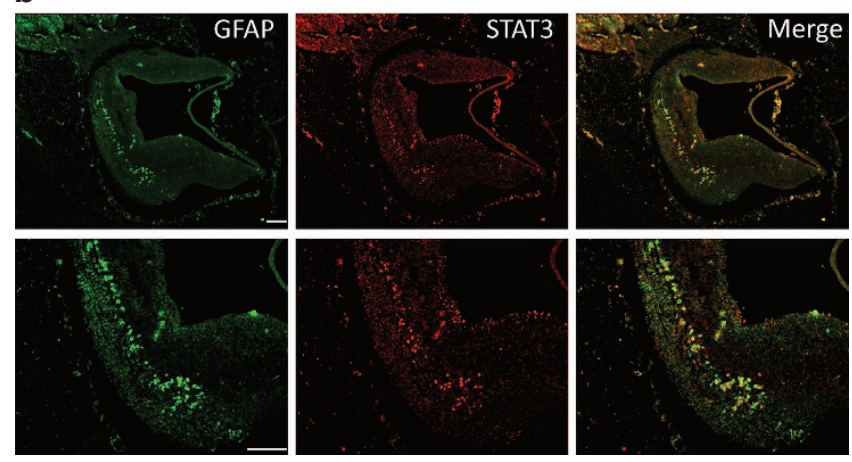

Figure 6. Expression of STAT3 in fetal brains. Micrographs of double immunostaining with a combination of either STAT3 and DCX antibodies or STAT3 and GFAP antibodies are shown in $\mathbf{a}$ and $\mathbf{b}$ respectively. Upper panels in both $\mathbf{a}$ and $\mathbf{b}$ micrographs show a low magnification of fetal brains while the lower panels show a high magnification of the fetal brains. Merged channels (Merge) show that STAT3 colocalizes with GFAP but not with DCX. Scale bar $=100 \mu \mathrm{m}$. DCX, doublecortin; GFAP, glial fibrillary acidic protein; STAT3, signal transducer and activator of transcription.

activators is still an open question. In addition to the potential negative impact of STAT3 in the disturbance of neurogenesis/ astrogenesis balance during prenatal development of the brain, injury-induced activated STAT3 in astrocytes have also been shown to exert beneficial effect on the proper maturation of oligodendrocytes cells during early neonatal life (26).

\section{Maternal Immune Stress and Corticosterone Levels in Fetal Brains}

In addition to proinflammatory cytokines, immune challenges also promote the synthesis and release of corticosterone (27). In normal conditions, the feto-placental enzyme $11 \beta$-hydroxysteroid dehydrogenase 2 shields fetuses from maternal GCs by inactivating maternal corticosterone (28). However, TLR-4-stimulated proinflammatory cytokines have the ability to alter the integrity of the placental barrier which could result in enhanced placental permeability to corticosterone (29). Thus, there is a possibility that maternally born corticosterone crosses the placenta and directly alters fetal brain development when the maternal immune system is activated (28,30). TLR-4 activation induced a significant increase in maternal plasma levels of corticosterone. These enhanced corticosterone levels were significantly reduced when the immune-challenged pregnant rats were given an

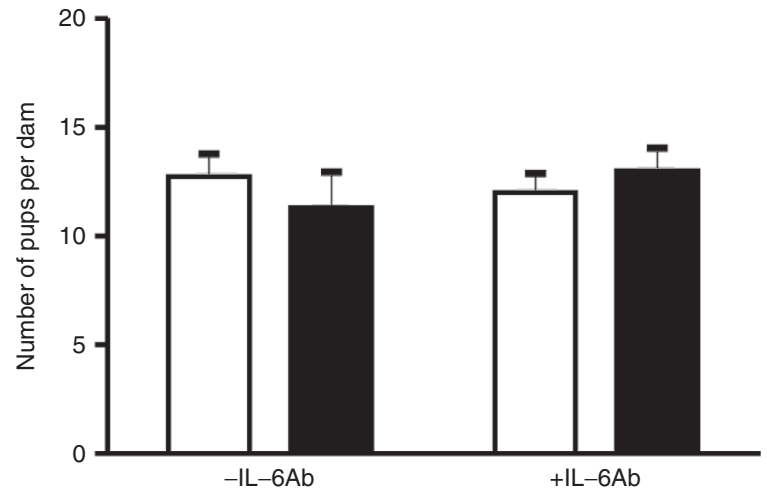

Figure 7. Impact of prenatal lipopolysaccharide (LPS) injection on litter size. GD15 rats were intraperitoneally injected with either saline (open bars) or LPS (solid bars) in the absence or the presence of IL6Ab. The number of living fetuses was counted $24 \mathrm{~h}$ after LPS injection. Injection of LPS and/or IL-6Ab did not significantly affect the litter size. Saline (-IL-6Ab; $n=$ 8), LPS (-IL-6Ab; $n=6)$, LPS (+IL-6Ab; $n=7)$, Saline (+IL-6Ab; $n=5)$.

IL-6 neutralizing antibody. This observation suggests that LPSinduced maternal corticosterone is, at least in part, mediated by IL-6. Indeed, IL-6 can directly stimulate corticosterone production from the adrenals (8).

We have noticed that maternal corticosterone response to LPS was larger at GD15 than that seen at GD16. Such enhanced responses were also seen in the pyrogen-free saline injected dams. These reduced responses at GD16 suggest that LPS effects is abated $24 \mathrm{~h}$ postinjection while the enhanced response to saline at GD15 is likely due to the stress of handling and injection. The reduced response at GD16 is less likely to be due to developmental changes as the maternal hyporesponsiveness to immune and emotional stressors occurs at the late-stage of pregnancy (31).

If maternal corticosterone crosses the placenta, one would expect it to be higher in the fetal compartment of immune challenged dams. Our data do not support such statement. In fact, corticosterone levels in fetal brains of dams given a febrile dose of LPS $(100 \mu \mathrm{g} / \mathrm{kg})$ (32) were not significantly different from those observed in fetal brains of dams given the pyrogenfree saline. This observation is in line with the role of placental barrier in inactivating maternal corticosterone and preventing its passage to the fetal compartment (33). Thus, the mild systemic inflammatory response does not appear to affect the placental barrier to GCs.

Maternal Immune Stress and Neural Cell Death in the Fetal Brain The activation of the maternal innate immune system with a febrile dose of LPS (32) leads to long lasting effects on brain plasticity and behavior of offspring during adulthood (4). This mild activation of the maternal innate immune response is associated with no overt deleterious effect on either pups survival (Figure 7 in the present paper) and (34) or neural cell death in the developing brain. However, administration of larger doses of LPS has been shown to enhance pups mortality and neural cell death in the brains of surviving pups (25). The absence of overt neural cell death in the fetal brains suggests that the long lasting impact of prenatal TLR4-mediated 
immune stress is probably induced through a rather subtle change in neuronal/glial imbalance. An imbalance which could be brought about by an enhanced activity of the IL-6STAT3 signaling pathway (24).

\section{METHODS}

\section{Animal Treatment}

Female Sprague-Dawley rats were mated with proven breeder male Sprague-Dawley rats and the vaginal plug was monitored daily. Positively identified females with vaginal deposition of sperm were housed individually. All rats were maintained at $22^{\circ} \mathrm{C}$ on $12 \mathrm{~h}$ light/ dark cycle (7 AM-7 PM), where food and water were available ad libitum. On GD15, rats were randomly separated into four groups and received the following i.p. injections: Group 1 received saline $2 \mathrm{~h}$ before LPS (100 $\mu \mathrm{g} / \mathrm{kg}$, from Escherichia coli; serotype 026:B6), group 2 received IL-6 neutralizing polyclonal antibody (IL-6Ab) dissolved in saline (R\&D systems, Minneapolis, MN, i.p. $10 \mu \mathrm{g} / \mathrm{kg}$ ) followed $2 \mathrm{~h}$ later with LPS $(100 \mu \mathrm{g} / \mathrm{kg})$, group 3 received IL-6Ab (i.p. $10 \mu \mathrm{g} / \mathrm{kg}$ ) followed $2 \mathrm{~h}$ later with saline, rat group 4 received two injections of saline in $2 \mathrm{~h}$ apart. All injections were performed on the morning (09:00 and 11:00). IL-6Ab is an IgG produced in goat and purified by rat IL-6 affinity chromatography. A different group of GD15 rats were given goat IgG in lieu of IL-6Ab to control for the specific effect of IL-6Ab. GD15 was chosen because TLR4 activation of the maternal immune system on GD15 is associated with altered brain function and plasticity of adult offspring (4). IL-6Ab was injected $2 \mathrm{~h}$ before LPS injection to allow for its neutralizing action before the immune insult (35). Each rat group includes at least five pregnant dams. From each dam only one male rat was used in either enzyme-linked immunosorbent assay (ELISA), fluorescent immunohistochemistry or western blot. These experiments were performed over a period of $2 \mathrm{y}$ and include data from three mating at different times of the year. All experiments were done in accordance with the guidelines on humane handling of experimental animals as established by the Canadian Council on Animal Care. The procedures employed were approved by the Animal Resources Centre of Kuwait University.

Rats were sacrificed at either 4 or $24 \mathrm{~h}$ post-LPS injection. These time point were chosen because $4 \mathrm{~h}$ post-LPS injection corresponds to the peak of STAT3 activation within the brain while $24 \mathrm{~h}$ was selected to assess the potential effect of LPS on neural cell death (36). We observed no significant impact of LPS or IL-6Ab injections on the litter size (Figure 7). The fetal brains were collected and snap frozen in liquid nitrogen and stored in the deep-freezer $\left(-80^{\circ} \mathrm{C}\right)$ until use in either ELISA or western blot studies. Maternal plasma and spleen were snap frozen in liquid nitrogen and stored at $-80{ }^{\circ} \mathrm{C}$ to assess the effect of IL-6Ab on LPS-activated maternal immune system and maternal corticosterone.

\section{Immunofluorescent Staining}

Whole fetuses were postfixed in 10\% neutral-buffered formalin solution, embedded in paraffin (Paraplast X-TRA, Sigma Aldrich, St. Louis, $\mathrm{MO})$ and processed for immunofluorescent staining as previously described (37). Serial thin sections $(5 \mu \mathrm{m})$ of paraffin embedded fetuses were mounted on microscope slides (Superfrost Plus, VWR, IL), rehydrated and a double immunofluorescent staining was performed. Neural cells committed to death were immunodetected using a polyclonal rabbit antibody anti-cleaved caspase 3 (1:1,000, Cell signaling MA) followed by an Alexa Fluor (A488)-tagged donkey anti-rabbit IgG $(1: 2,000,2 \mathrm{~h}$ at room temperature; life technologies, CA). For cell count, slides were re-coded to allow for blind counting. Images of at least ten randomly selected fetal brain areas were taken using a $20 \times$ objective (Axio Imager A1, Carl Zeiss Microscopy GmbH/Germany) and positively stained cells were counted. Cleaved caspase 3 were counted both at the parenchymal level of the fetal brain (inside) and at the meningeal edge of the brain (edge). The data for the number of cleaved caspase- 3 containing cells in the "inside" part of the fetal brain are presented as number of cells per area in $\mathrm{mm}^{2}$. Cleaved caspase- 3 containing cells in the "edge" part of the fetal brain were counted, a line was drawn on the meningeal edge of brain, its length was measured and the data was presented as the number of cells per line length in $\mathrm{mm}$.

In a different series of experiments, a double immunofluorescent staining was performed. Rehydrated fetal brain sections were incubated (overnight at room temperature) with a polyclonal rabbit antibody anti-STAT3 (1:1,000, Santa Cruz Biotechnology, CA) in combination with either a polyclonal goat antibody anti-doublecortin $(1: 1,000$, Santa Cruz biotechnology) or a monoclonal mouse antibody anti glial fibrillary acidic protein (1:1,000, Sigma Aldrich). After washing, fetal brain sections were incubated with a combination of Alexa Fluor (A488 or A555)-tagged secondary antibodies (donkey anti-rabbit IgG (for STAT3 detection) and donkey anti-goat IgG (for doublecortin detection) or donkey anti mouse IgG (for glial fibrillary acidic protein detection) (all at 1:2,000, life technologies, CA).

\section{Western Blot}

Proteins of maternal spleens were extracted and separated using a $12 \%$ SDS-PAGE. Proteins were then transferred to a nitrocellulose membrane and incubated with a mouse monoclonal antibody anti-rat phospho-STAT3 (1:1,000, Cell Signaling Technology, Danvers, MA) for $16 \mathrm{~h}$ at $4{ }^{\circ} \mathrm{C}$. The nitrocellulose membranes were washed in TBS-T solution (Trizma-base ( $20 \mathrm{mmol} / \mathrm{l}), \mathrm{NaCl}(150 \mathrm{mmol} / \mathrm{l})$, and Tween 20 $(0.1 \%))$ and were exposed to a horse radish peroxidase tagged secondary antibody anti-mouse IgG (1:2,000, Santa Cruz Biotechnology, TX) for $2 \mathrm{~h}$ at room temperature. Bound antibodies were revealed using an enhanced chemiluminescence assay (GE Healthcare UK, England). After detection of phospho-STAT3, the membrane-bound antibodies were removed with 2-mercaptoethanol (Sigma Aldrich) and the membrane was reexposed to a primary polyclonal rabbit antibody anti-total STAT3 (1:1,000, Santa Cruz Biotechnology, CA) followed by a horse radish peroxidase tagged secondary antibody anti rabbit $\operatorname{IgG}(1: 2,000$, Santa Cruz Biotechnology) as previously described (38).

\section{ELISA}

ELISA was used to measure corticosterone, IL-6 and phospho-STAT3 levels. Maternal heads were rapidly decapitated with a guillotine and maternal trunk blood samples were collected. Blood samples were collected in the morning (9:00-11:00). The maternal plasma samples were collected by centrifugation, snap-frozen in liquid nitrogen, and stored in a $-80{ }^{\circ} \mathrm{C}$ freezer. Plasma levels of either corticosterone or IL-6 were assayed using a corticosterone EIA kit (Cayman Chemicals, Ann Arbor, MI) and a rat IL-6 ELISA kit respectively (Invitrogen, Camarillo, CA). The sensitivity of corticosterone EIA kit is $30 \mathrm{pg} / \mathrm{ml}$ with an intra-assay variability of $5.6-20.3 \% \mathrm{CV}$ and inter-assay variability of $3.8-22.5 \% \mathrm{CV}$. The sensitivity of rat IL-6 ELISA is $<5 \mathrm{pg} / \mathrm{ml}$ with an intra-assay variability of $2.8-5.8 \% \mathrm{CV}$ and inter-assay variability of 5.7-8.8 \%CV. Fetal brains were collected, snap frozen in liquid nitrogen and stored in $\mathrm{a}-80^{\circ} \mathrm{C}$ freezer. Fetal brains were homogenized and levels of phospho-STAT3 and corticosterone were measured using either corticosterone or phospho-STAT3 (Thermo Scientific Pierce, Rockford, IL) ELISA kits as previously described (39). Levels of total proteins in each sample were assessed colorimetrically using a BCA Protein Assay Kit (Thermo Scientific Pierce). The ELISA data are presented as the amount of analyte (corticosterone or phospho-STAT3) per mg of protein. The Phospho-STAT3 ELISA kit detects specifically STAT3 phosphorylated at tyrosine residue 705 with the following characteristics: interassay variability, $2.56-7.22 \% \mathrm{CV}$; intra-assay variability, 2.1-6.13\%CV; and a sensitivity of less than 0.9 Units $/ \mathrm{ml}$.

\section{Statistics}

For densitometry analysis of western blots, the area under the intensity profile curve of a given band was quantified using ImageJ software (40). All values were calculated as a ratio of p-STAT3/totalSTAT3 or cleaved caspase 3/actin, expressed as a fold change of the values in animals that received saline only. All data (western blot, immunostaining cell counts, ELISA) were compared using two-way ANOVA followed by Student-Newman-Keuls post-hoc test whenever possible. The number " $\mathrm{n}$ " in the data related to the offspring corresponds to the number of individuals derived from " $\mathrm{n}$ " different litters. The difference between groups was declared statistically significant at $P<0.05$. 


\section{ACKNOWLEDGMENTS}

The authors thank S. Kalakh and Beena Sebastian for their help with animal husbandry and technical support and Dr. Maie D. Al Bader for the critical reading of this manuscript.

\section{STATEMENT OF FINANCIAL SUPPORT}

This work was supported by Kuwait University Research Grant MY01/09 to A.M. Imaging was performed in the Research Unit for Genomics, Proteomics and Cellomics Sciences supported by Research Project No. SRUL02/13.

Disclosure: There is no conflict of interest to disclose.

\section{REFERENCES}

1. Khandaker GM, Zimbron J, Lewis G, Jones PB. Prenatal maternal infection, neurodevelopment and adult schizophrenia: a systematic review of population-based studies. Psychol Med 2013;43:239-57.

2. Khan D, Fernando P, Cicvaric A, et al. Long-term effects of maternal immune activation on depression-like behavior in the mouse. Transl Psychiatry 2014;4:e363.

3. Mouihate A. Long-lasting impact of early life immune stress on neuroimmune functions. Med Princ Pract 2013;22 Suppl 1:3-7.

4. Boksa P. Effects of prenatal infection on brain development and behavior: a review of findings from animal models. Brain Behav Immun 2010;24: 881-97.

5. Ashdown H, Dumont Y, Ng M, Poole S, Boksa P, Luheshi GN. The role of cytokines in mediating effects of prenatal infection on the fetus: implications for schizophrenia. Mol Psychiatry 2006;11:47-55.

6. Dahlgren J, Samuelsson AM, Jansson T, Holmäng A. Interleukin-6 in the maternal circulation reaches the rat fetus in mid-gestation. Pediatr Res 2006;60:147-51.

7. Harré EM, Mouihate A, Pittman QJ. Attenuation of fever at near term: is interleukin-6-STAT3 signalling altered? J Neuroendocrinol 2006;18:57-63.

8. Päth G, Scherbaum WA, Bornstein SR. The role of interleukin-6 in the human adrenal gland. Eur J Clin Invest 2000;30 Suppl 3:91-5.

9. van der Meer MJ, Sweep CG, Rijnkels CE, et al. Acute stimulation of the hypothalamic-pituitary-adrenal axis by IL-1 beta, TNF alpha and IL-6: a dose response study. J Endocrinol Invest 1996;19:175-82.

10. Zacharowski K, Zacharowski PA, Koch A, et al. Toll-like receptor 4 plays a crucial role in the immune-adrenal response to systemic inflammatory response syndrome. Proc Natl Acad Sci USA 2006;103:6392-7.

11. Maccari S, Darnaudery M, Morley-Fletcher S, Zuena AR, Cinque C, Van Reeth O. Prenatal stress and long-term consequences: implications of glucocorticoid hormones. Neurosci Biobehav Rev 2003;27:119-27.

12. Paintlia MK, Paintlia AS, Contreras MA, Singh I, Singh AK. Lipopolysaccharide-induced peroxisomal dysfunction exacerbates cerebral white matter injury: attenuation by N-acetyl cysteine. Exp Neurol 2008;210: $560-76$.

13. Paintlia MK, Paintlia AS, Barbosa E, Singh I, Singh AK. N-acetylcysteine prevents endotoxin-induced degeneration of oligodendrocyte progenitors and hypomyelination in developing rat brain. J Neurosci Res 2004;78:347-61.

14. Yakovlev AG, Ota K, Wang G, et al. Differential expression of apoptotic protease-activating factor-1 and caspase- 3 genes and susceptibility to apoptosis during brain development and after traumatic brain injury. J Neurosci 2001;21:7439-46.

15. Meyer U, Feldon J, Yee BK. A review of the fetal brain cytokine imbalance hypothesis of schizophrenia. Schizophr Bull 2009;35:959-72.

16. Zaretsky MV, Alexander JM, Byrd W, Bawdon RE. Transfer of inflammatory cytokines across the placenta. Obstet Gynecol 2004;103:546-50.

17. Urakubo A, Jarskog LF, Lieberman JA, Gilmore JH. Prenatal exposure to maternal infection alters cytokine expression in the placenta, amniotic fluid, and fetal brain. Schizophr Res 2001;47:27-36.
18. Wagner RK, Hinson RM, Apodaca CC, et al. Effects of lipopolysaccharide on interleukin-6 production in perfused human placental cotyledons. J Matern Fetal Med 2000;9:351-5.

19. Ghiani CA, Mattan NS, Nobuta H, et al. Early effects of lipopolysaccharide-induced inflammation on foetal brain development in rat. ASN Neuro 2011;3:

20. Nakashima $K$, Yanagisawa $M$, Arakawa $H$, et al. Synergistic signaling in fetal brain by STAT3-Smad1 complex bridged by p300. Science 1999;284:479-82.

21. Meyer U. Prenatal poly(i:C) exposure and other developmental immune activation models in rodent systems. Biol Psychiatry 2014;75:307-15.

22. Smith SE, Li J, Garbett K, Mirnics K, Patterson PH. Maternal immune activation alters fetal brain development through interleukin-6. J Neurosci 2007;27:10695-702.

23. Yoshimatsu T, Kawaguchi D, Oishi K, et al. Non-cell-autonomous action of STAT3 in maintenance of neural precursor cells in the mouse neocortex. Development 2006;133:2553-63.

24. Taga T, Fukuda S. Role of IL-6 in the neural stem cell differentiation. Clin Rev Allergy Immunol 2005;28:249-56.

25. Galic MA, Riazi K, Heida JG, et al. Postnatal inflammation increases seizure susceptibility in adult rats. J Neurosci 2008;28:6904-13.

26. Nobuta H, Ghiani CA, Paez PM, et al. STAT3-mediated astrogliosis protects myelin development in neonatal brain injury. Ann Neurol 2012; 72:750-65.

27. Beishuizen A, Thijs LG. Endotoxin and the hypothalamo-pituitary-adrenal (HPA) axis. J Endotoxin Res 2003;9:3-24.

28. Mouihate A, Al-Bader MD. Glucocorticoid-induced fetal brain growth restriction is associated with p73 gene activation. J Neurosci Res 2013;91:95-104.

29. Kirsten TB, Lippi LL, Bevilacqua E, Bernardi MM. LPS exposure increases maternal corticosterone levels, causes placental injury and increases IL-1B levels in adult rat offspring: relevance to autism. PLoS One 2013;8:e82244.

30. Singh RR, Cuffe JS, Moritz KM. Short- and long-term effects of exposure to natural and synthetic glucocorticoids during development. Clin Exp Pharmacol Physiol 2012;39:979-89.

31. Mouihate A, Harré EM, Martin S, Pittman QJ. Suppression of the febrile response in late gestation: evidence, mechanisms and outcomes. J Neuroendocrinol 2008;20:508-14.

32. Mouihate A, Abdeslam M. Prenatal immune stress in rats dampens fever during adulthood. Dev Neurosci 2012;34:318-26.

33. Chapman K, Holmes M, Seckl J. 11ß-hydroxysteroid dehydrogenases: intracellular gate-keepers of tissue glucocorticoid action. Physiol Rev 2013;93:1139-206.

34. Cui K, Ashdown H, Luheshi GN, Boksa P. Effects of prenatal immune activation on hippocampal neurogenesis in the rat. Schizophr Res 2009;113:288-97.

35. Rummel C, Sachot C, Poole S, Luheshi GN. Circulating interleukin-6 induces fever through a STAT3-linked activation of COX-2 in the brain. Am J Physiol Regul Integr Comp Physiol 2006;291:R1316-26.

36. Yamawaki Y, Kimura H, Hosoi T, Ozawa K. MyD88 plays a key role in LPS-induced Stat3 activation in the hypothalamus. Am J Physiol Regul Integr Comp Physiol 2010;298:R403-10.

37. Spencer SJ, Galic MA, Tsutsui M, Pittman QJ, Mouihate A. Effects of global cerebral ischemia in the pregnant rat. Stroke 2008;39:975-82.

38. Mouihate A, Galic MA, Ellis SL, Spencer SJ, Tsutsui S, Pittman QJ. Early life activation of toll-like receptor 4 reprograms neural anti-inflammatory pathways. J Neurosci 2010;30:7975-83.

39. Mouihate A. TLR4-mediated brain inflammation halts neurogenesis: impact of hormonal replacement therapy. Front Cell Neurosci 2014;8:146.

40. Schneider CA, Rasband WS, Eliceiri KW. NIH Image to ImageJ: 25 years of image analysis. Nat Methods 2012;9:671-5. 